\title{
Steps to Development and Increase of Physical Movement and Health Literacy
}

M. Rafajdus (Michal Rafajdus), M. Machajova (Michaela Machajova)

Trnava University, Faculty of Health Sciences and Social Work, Department

Original Article of Public Health, SK.

\section{E-mail address:}

michal.rafajdus@truni.sk

\section{Reprint address:}

Michal Rafajdus

Department of Public Health

Faculty of Health Sciences and Social Work

Trnava University in Trnava

Univerzitne nam. 1

91843 Trnava

Slovakia

Source: Clinical Social Work and Health Intervention

Pages: $66-71$

Volume: 12

Issue: 5

\section{Reviewers:}

Gabriela Lezcano

University of California, San Francisco, USA

Vitalis Okoth Odero

Catholic university of Eastern Africa, Nairobi, KE

\section{Keywords:}

Knowledge. Lifestyle. Physical Movement Literacy. SM System.

\section{Publisher:}

International Society of Applied Preventive Medicine i-gap

CSWHI 2021; 12(5): 66 - 71; DOI: 10.22359/cswhi_12_5_10 (C) Clinical Social Work and Health Intervention

\section{Abstract:}

According to the WHO, health is a state of complete physical, mental and social well-being while maintaining the body's ability to adapt to changing environmental conditions. The definition is concise, but it is important to be aware of the essential facts that affect people of the 21 st century. Is it possible to adapt to rapidly changing environmental conditions? Musculoskeletal conditions are considered a global epidemic as their incidence and severity increase worldwide. Consideration should be given to the fact that musculoskeletal conditions, such as back pain (diagnosis of M54: dorsalgia), are beginning to be epidemiological in developed countries. According to the Institute for Health Metrics and Evaluation, the most common causes of years lived with disability (YLDs) in the Slovak Re- 
public from 2005 to 2015 were non-communicable diseases. In 1st place were musculoskeletal deformities; 2nd place mental disorders and addictions; $3^{\text {rd }}$ place other unspecified noninfectious diseases (Source: GBD, 2015). It is important to tackle this issue not only because of its permanent growth but especially for the frequent complications that cause higher morbidity. Various postural deformities caused by poor posture, which affect the relationships between internal organs and the musculoskeletal system/viscero-vertebral relationships but also vice versa. The prevalence of musculoskeletal diseases has an increasing trend in Slovakia, and therefore it is important to look for a way to raise awareness of the possibilities of these diseases' prevention. It is essential to revise outdated plans for physical development and adapt them to today's people. After all, the movement literacy of our children has a significantly declining tendency. Such intervention gives meaning to the public health; there should be a focus on the primary support of the health of the population where one of the possibilities may be the spiral mobilization method SM system. This method is intended for a person living in current times, who sits to a large extent and performs movements that are monotonously loading the muscles. The SM system is a simple and effective exercise that on the principle of spiral muscle chains pulls the spine upwards and stabilizes the balance of the spine. When performed in an optimal way, they relax overloaded parts of the spine; reduce muscle imbalance; contribute to overall health. The priority for proper physical movement should be a desire for a healthy body without pain and disease. The path to health leads through cognition and education because the most important result of any education is self-knowledge.

\section{Introduction}

"Health, health, health" - we believe that this is the most important thing we have. The current questions are: Is that really so? Are we trying to do something for our own health? Or are they just common phrases?

According to the WHO, health is a state of complete physical, mental and social well-being while maintaining the body's ability to adapt to changing environmental conditions. The definition is concise, but it is important to be aware of the essential facts that affect people of the 21 st century. Technology is advancing by leaps and bounds, but we humans and our genetics, which have evolved over millennia, cannot change as fast as we imagine and thus today's modern man is paying for their current lifestyle. From our own professional practice, we do notice that people are not aware of this change and do not admit it. We crave for knowledge, but we have forgotten the legacy of the ancient Greeks that the development of mental abilities should be harmoniously connected with all-round physical development and vice versa. This means that physical movement and knowledge move hand in hand to health.

\section{Habits are Formed in Infancy}

The instinctive physical key movement habits have their origins in the process of physical development initiated in the first days of baby's life. The newborns wiggle and twist on their stomachs which are movements that precede crawling and they push against the mat on which they are lying - whether it's their mother's body, a mattress in a crib, or a blanket on the floor. Such movements form the basis on which a naturally upright and healthy body will be built. Not only do these movements provide extensive stimulation of sensory and motor neurons that map the connections 
between the body and the brain, but they also build a firm, strong center of the body that ensures and maintains body's structural integrity.

As the baby's inner muscles in the middle of the body strengthen, it also awakens its ability to complete a series of naturally evolving developmental milestones, such as rolling over and sitting, and then climbing and sitting on its side, building each task on the previous one, until the child reaches the peak of the primary human goal - uprightness. And once the baby manages to find balance while standing on both lower limbs, they will stand, walk and run forever. Once a well-developed child can stand and walk, their body center is strong enough to stabilize their spine and, at first attempts, control their body with their own energy. Much more important than whether this happens sooner or later is whether the child has been given the opportunity to actively participate in these neurodevelopmental movements in order to build a healthy nervous system and a firm and strong chain of body muscles which will provide natural support for the upright body in the upcoming years.

Babies who do not explore and do not undergo progressive developmental movements may never build a strong enough foundation to fix the natural position of the pelvis, which provides a comfortable support for the upright spine. Although such children are still moving and are achieving other developmental goals, which present the ability to learn to stand, walk and run, they have probably already adopted adaptive movement patterns that will function as new and unnatural basic setting. The weak center of the body, the oppressed spine and the general structural axis deviation will negatively affect their health and vitality in the following years.

\section{Current Standard}

Many chronic health problems that are nowadays common not only among Americans can be avoided or solved by better diet; increased physical activity; reduced stress. What is missing from the list of recognized causes of various health problems is the incorrect position of the whole structure. Poor posture is an important factor that plays a crucial role in the large number of cases of chronic pain experienced by millions of people. Until we understand the details, natural biomechanical forms of the body and acknowl- edge their importance, and until they become part of medical school education, the pain epidemic and other posture health problems will not stop afflicting millions of adults and children around the world.

Although poor posture has become an increasing problem since people began spending more time at their desks, in their cars and in front of the television, it cannot be compared to the drastic changes that have taken place since the intensive use of computers, mobile phones and other digital devices which claim more and more time from our daily lives. Today, poor posture can be seen even in babies and young children. The fear of placing babies on their stomachs, the often-repeated rule that a baby must lie on their backs, and the growing dependence on various positioning aids for infants have led to too many babies spending almost all of their time, whether awake or asleep, either lying on their backs or leaning in loungers. As a result, their weight is constantly transferred to the back of the pelvis, often in a collapsed position in baby car seats, rocking chairs, prams, carriers or swings. All of this distorts their inner abdominal muscles, which then weaken. The more we know the role of specific physical movements in healthy development, the clearer we will see how unhappy this trend is.

\section{Increased Incidence of Disorders in Children}

At the same time, school teachers, occupational therapists, physiotherapists and other experts in what is described as a child's normal development observe: an increasing number of children who show chronically collapsed posture; reduced muscle tension; difficulty with balance and coordination; ankles valgus; flat feet or collapsed arch; acquired hip dysplasia; unintegrated primitive reflexes; vision problems; behavioral disorders; multiple food allergies and intolerances; chronic digestive problems; sleep disorders; apathy and fatigue, anxiety; depression ... - and the list goes on (Porter, 2017).

Why are we witnessing such a shocking increase in the incidence of disorders, diseases and health problems in our children? Changes made to diagnosis criteria and case reporting processes may play a role, but they are not in themselves sufficient to explain the overwhelming nature of this phenomenon. Many genetic and environ- 
mental factors are also likely to contribute to the status quo. In conjunction with each other, some then create complex interactions, for example when an existing genetic risk "meets" certain environmental factors. Suppression of neurodevelopmental movement in children is one of the key factors that may worsen the innate predisposition to develop: autism; attention-deficit disorders; other health problems; and may also be the primary cause itself.

Although parents, teachers and health professionals may be aware that today's children often are prone to a hunched posture, there are no effective measures to tackle this issue. This is because our society does not understand what natural really is (a collapsed posture, reduced muscle tension and problems with coordination and balance), what then determines which characteristics prevail - whether natural or unnatural?

An upright figure aligned along the axis: what it looks like; how it works; how it manifests itself in the correct posture. When the naturally upright figure is characterized by a firm posture aligned with the body core, effortlessly engaged and noticeably relaxed in its balance and coordination it is characterized by the "big three" - (body axis, muscle balance, muscle range). It is important to note that anyone, of any age, is able to return to a naturally upright posture. Many people, including myself, have been able to successfully overcome chronic tension and pain and improve and overcome a long list of other health problems that may have occurred later in life.

People can ignore the fact that they come from nature or forget about it, but we are still dependent on it. Our health and quality of life are largely determined by the degree of our compliance with the laws of nature. From the moment of conception and further in the mother's uterus, a new life cycle begins to be written - a continuous run from birth, through childhood, adulthood, old age and finally to death, accompanied by a pattern that nature has given us.It is tragic that the violation of the laws of nature is now causing problems to the youngest of our species - our babies and children. While some difficulties arise for reasons we are unable to understand or control, many developmental "disorders" appear to be caused by factors that are in our power to influence if we understand how the collective "we "involuntarily violates certain laws of nature.

\section{Physical Literacy as a Way to Health}

We need to give consideration to the fact that musculoskeletal problems, such as back pain (diagnosis of M54: dorsalgia), are beginning to be epidemiological in developed countries. According to the Institute for Health Metrics and Evaluation, the most common causes of "years lived with disability" (YLDs) in the Slovak Republic from 2005 to 2015 were non-communicable diseases. In 1st place were musculoskeletal deformities; $2^{\text {nd }}$ mental disorders and addictions; $3^{\text {rd }}$ other unspecified non-infectious diseases (GBD, 2015). According to information from the Social Insurance Agency of the Slovak Republic, disability due to diseases of the musculoskeletal system in Slovakia for the years 1997 - 2016 has highly increasing character (SP, 2016).

It is important to address this issue, not only because of its permanent growth, but especially for the frequent complications that cause higher morbidity. Various postural deformities arising from poor posture, which affects the relationships between internal organs and the musculoskeletal system/viscera-vertebral patterns/, but also vice versa. Also breathing which is the basis for the correct position of the body and the exchange of gases in the body. All of this is a physiological need for health, but the modern age brings us psychological strain, which is also reflected in posture and breathing habits. Due to the growing prevalence of musculoskeletal disorders in the Slovak Republic, it is important to look for an effective way of prevention. One option is to raise awareness of this issue.

Organization of mass sports events, such as marathons, has become a phenomenon of recent years. On the one hand, enthusiasm for physical movement is welcome, but on the other hand, physical movement can also be detrimental to health for those who do not perform it properly. Our lifestyle, consisting of: sitting; driving; stress; unbalanced diet; smoking, leads to degeneration of the musculoskeletal system especially of the spine. Once we decide to start a sports activity, we must realize that we are not healthy if we perform it a lot, but if we do it adequately, correctly and consciously. Three principles are essential for proper movement: proper functional body axis; muscle balance (all muscles have sufficient and even development); muscle and movement range of motion. Also, exercise with 
one-sided sports load must include corrective and compensating elements. These must be monitored to re-create the correct axial position. Otherwise, unilateral exercise-sports load can cause the development of other muscle imbalances. All this is a need that brings longevity into sport. With one-sided, unconscious loading of the body, postural imbalances and health problems quickly arise. Our body is made up of about 600 muscles. Therefore, our emphasis must be on adequate comprehensive development, not just on esthetics, nor on superficial muscles. We have genetically determined proper movement habits, and therefore our proper movement development is influenced by a large number of factors since birth. Modern times have a very negative impact on proper physical movement habits. It is important to perceive the physical movement because with conscious and correct body movement, pathological movement habits can be eliminated.

\section{SM System Method}

Physical literacy of our children, with markedly declining trend, serves as a reflection of the current times. Therefore, in addition to the curriculum, preventive and compensatory exercises should be included in the everyday life of students and their families. A child spends most time in domestic and work environment observing their family members, friends or teachers. At school, a teacher should be able to correct and admonish the child (pupil), at home this responsibility is left to the parents. The intention is to build the right habits when sitting, standing, walking or performing any physical activity. A recommended way of preventive exercises is the so-called SM-system, which is physically and financially undemanding. At the same time, in practice, it is a proven way to prevent various musculoskeletal problems.

The SM system method, i.e. the "Stabilization and Mobilization System", is based on 30 years of gradual development and 25 years of clinical experience with this exercise in patients with spinal, thoracic and cervical back pain; in patients with acute disc disease; scoliosis. This exercise eliminates both, local and general causes of back pain at the same time. It is of great importance in the prevention and treatment of joint disorders (hip, knee, shoulder, leg and arch joints) (Smisek, 2013).
The SM system provides functional stabilization and mobilization of the spine. This method works on the principle of spiral muscle chains that pull the spine upwards and at the same time stabilize the balance of the spine. When performed in an optimal way, they relax overloaded parts of the spine and strengthen weakened muscles which reduces muscle imbalance. It is performed with an elastic rope that is attached to a fixed point which allows extensive movement of the limbs against a small and gradually increasing resistance. This activates the muscle spirals with a stabilizing function. The elastic rope can be understood as an extension of the muscle fibers that activate these spirals. Exercise allows some muscles to stretch during a time of natural relaxation and at the same time strengthen others thanks to elastic resistance. Each rope has a loop at the end that is worn on a hand, similar to a running stick. It is only set aside in the hand. Incorrect way of holding it is a crampy grasp that overloads the wrist and elbow, and which can lead to carpal tunnel syndrome or lateral/medial epicondylitis (tennis elbow syndrome). Gentle grip also results in greater loosening of the neck tension. In the case of the lower limb, we put this loop on a foot (Smisek, 2013). Exercise SM system combines important components: movement and its optimal coordination; muscular apparatus and formation of descending muscle spirals; the response to the spine which is the centring (i.e. alignment to the midline and traction, i.e. upward stroke in the whole spine or only in certain segments (Smisek, 2013).

There are several principles that need to be followed when exercising. 1st is the exercise position. It is practiced in standing position; the body must be aligned along the axis given by the earth's gravity. However, the axis may deviate slightly to compensate for the elastic force of the rope. The 2 nd principle is the alternation of activation (spiral stabilization) and relaxation (vertical stabilization) along with inhaling and exhaling. $3^{\text {rd }}$ the stand must be balanced and the body strengthened at a time when force is acting on it. At a time when no force is acting on the body, we are completely relaxed. 4th is a coordination of the movement, the strengthening of the body is performed from the bottom up which means from the pelvis (switching on the gluteal muscles) to the shoulder girdle and continue to a balanced 
position of the head. We relax from the nape downwards. The strength with which one exercises is another part. It is a small force in the widest possible range of motion and it respects the weakest link in the chain of motion. 5th principle is the speed of exercise - the exercise is performed slowly. At the end of the movement, we slow down even more so that we can finish the final detail. The movement is slow and smooth, we do not perform it jerkily. It is also important to focus on the complexity of the movements. If the exercise is performed adequately, the muscles from the surface of the foot to the fingers of the hands are activated. The choice of exercises is also very important. First, the symmetrical exercises are preferred, which fix muscle imbalances, followed by asymmetrical exercises, moving from simpler exercises (on both lower limbs) to more complex ones (only on one, standing limb) (Smisek, 2013). This method is intended for a person living in current times, who sits to a large extent and performs movements monotonously loading the muscles.

The importance of public health lies in the orientation to support the health of the population where one of the possibilities is the exercise of the spiral-mobilization method SM system as well as properly designed educational lectures by an expert. This method is intended for a person living in current times who sits to a large extent and performs movements that are monotonously loading the muscles. The SM system is a simple and effective exercise that, on the principle of spiral muscle chains pulls the spine upwards and stabilizes the balance of the spine. When performed in an optimal way they: relax overloaded parts of the spine; reduce muscle imbalance; contribute to overall health. The priority for proper physical movement should be a desire for a healthy body without pain and disease.

\section{Movement Hygiene and Literacy}

Movement hygiene and literacy can help people find and use information and strengthen the impact on their own health. It is important that health literacy becomes part of the upbringing of children. The sooner a child acquires the necessary knowledge, skills and habits and the sooner we manage to contribute to the formation of their attitudes, the greater the benefit of health literacy for their health. The development of health liter- acy increases the overall level of the health of the population. The main benefit of health literacy is that it helps people improve their health. Health is the most important and crucial value of every person, as well as of society as a whole and that is why building and mastering health literacy should be an integral part of our lives. Through health literacy the citizen becomes an active factor in the healthcare system. Their decision can be based on proven knowledge about health problems and about the ways of solving them. With their attitudes, interests and needs, people can influence the further development of the healthcare system, and thus the favorable development of the population's health.

\section{Conclusion}

The path to health leads through cognition and education because the most important result of any education is self-knowledge. From our experience, it is about the people's knowledge and awareness of a given posturological issue (posturology is the science of posture). After each lecture and consultation, people are surprised at the amount of new information they have learned and wonder why this is not being talked about in education, healthcare or sports. Probably because it wasn't necessary in earlier times. Lifestyle did not use to have such a serious impact on health, and therefore, nowadays, this new role is relevant for trainers, teachers, health professionals and therapists. It is essential to revise outdated plans for physical development and adapt them to today's people. Exercise is like medicine, but we need to know how to dose it properly to achieve its effect. Such intervention gives meaning to the public health - a focus on the primary support of the health of the population.

\section{References}

1. GLOBAL BURDEN OF DISEASE (GBD) (2015).

2. PORTER K (2017) Healthy posture for babies and children: tools for helping children to sit, stand, and walk naturally. Rochester, Vermont: Healing Arts Press.

3. SMISEK, R. et al. 2013. Healthy back. 1. ed. Praha: Richard Smisek, 2013. p. 173. ISBN 9788087568200.

4. SOCIAL INSURANCE AGENCY OF THE SLOVAK REPUBLIC (2016). 